J. theor. Biol. (1974) 47, 209-221

\title{
The Theory of Games and the Evolution of Animal Conflicts
}

\begin{abstract}
J. Maynard Smith
School of Biological Sciences, University of Sussex, Falmer, Brighton, Sussex BN1 9QG, England

(Received 10 January 1974)

The evolution of behaviour patterns used in animal conflicts is discussed, using models based on the theory of games. The paper extends arguments used by Maynard Smith \& Price (1973) showing that ritualized behaviour can evolve by individual selection. The concept of an evolutionarily stable strategy, or ESS, is defined. Two types of ritualized contests are distinguished, "tournaments" and "displays"; the latter, defined as contests without physical contact in which victory goes to the contestant which continues longer, are analyzed in detail. Three main conclusions are drawn. The degree of persistence should be very variable, either between individuals or for the same individual at different times; a negative exponential distribution of persistence times is predicted. Individuals should display with constant intensity, independent of how much longer they will in fact continue. An initial asymmetry in the conditions of a contest can be used to settle it, even if it is irrelevant to the outcome of a more protracted conflict if one were to take place.
\end{abstract}

\section{Introduction}

Most models of evolution ascribe "fitnesses" to individuals and then work out the way in which the frequencies of individuals of various kinds in the population change with time. Sometimes these fitnesses are assumed to be constant; sometimes it is supposed that the environment and hence the relative fitnesses of different genotypes change either in space or time. Such models are quite satisfactory for many purposes. In recent years, however, there has been increasing interest in the evolution of characteristics which cannot adequately be analyzed by such models, for one or more of the following reasons:

(1) The characteristic affects the survival of populations differently from that of individuals; examples are sexual reproduction (Williams, 1966; Williams \& Mitton, 1973; Maynard Smith, 1971) and characteristics serving to regulate population density (Wynne Edwards, 1962). 
(2) The characteristic affects the survival or reproduction of relatives of the individual possessing it (Hamilton, 1964; Trivers, 1972).

(3) The fitness of a particular genotype depends on what other genotypes are present in the population and on their frequencies.

The corresponding selective mechanisms in these three situations are group selection, kin selection and frequency-dependent selection. All three have been proposed as explanations for the comparative rarity of dangerous weapons or tactics in intra-specific animal conflicts. Thus Huxley (1956) probably expressed the most commonly held view when he argued that the use of dangerous weapons is rare because "it would militate against the survival of the species". Hamilton (1971) has emphasized the evolutionary importance of the fact that excessively aggressive individuals may injure their close relatives. Maynard Smith \& Price (1973) proposed a model of the evolution of conflict behaviour in which selection acts entirely at the individual level, but in which the success of any particular strategy depends on what strategies are adopted by other members of the population. The purpose of this paper is to pursue this line of reasoning a little further, particularly in the case in which serious injury is impossible.

The main conclusion reached by Maynard Smith \& Price (1973) was that in a species capable either of "ritualized" or "escalated" fighting-the latter being capable of seriously injuring an opponent-the evolutionarily stable strategy is to adopt the ritualized level, but to respond to escalation from an opponent by escalating in return. The importance of retaliation in the evolution of animal conflict was emphasized earlier by Geist (1966). In a population adopting such a "retaliation" strategy, a mutant which adopted escalation tactics too readily would be more likely to get seriously injured than the typical members of the population, who would usually settle conflicts without escalation. This conclusion, however, rested on the assumption that two individuals adopting purely ritualized methods could satisfactorily settle a contest. This assumption is investigated further in this paper.

Two types of ritualized contest must be distinguished, which I will call "tournaments" and "displays". An example of a tournament is a fight between two male deer, in which the antlers interlock and a pushing match ensues. The structure of antlers and the behaviour of the contestants is adapted to prevent serious injury. Physical contact does take place, however, and victory goes to the larger, stronger and healthier individuals. Tournaments of this kind are common. In such cases, no special difficulty arises in understanding how a ritualized contest can be settled; the model considered by Maynard Smith \& Price (1973) seems adequate to explain why more dangerous weapons or tactics do not evolve. 
In a "display", no physical contact takes place, or if it does do so it does not settle the contest and provides little or no information about which contestant would win an escalated contest. In such a contest, the winner is the contestant who continues for longer, and the loser the one who first gives way. It is the logic of contests of this kind that is considered in this paper. From an evolutionary point of view, tournaments and displays have something in common, in that the winner is the individual which continues the contest longer, and we would therefore expect natural selection to favour characteristics (size and strength in one case and behavioural persistence in the other) enabling an individual to continue; there would be an ultimate balance between the advantages and disadvantages of increased size or persistence. There are, however, important differences. First, size and strength may change with age, but cannot be varied from day to day or from contest to contest. Second, the corresponding disadvantages of greater size would arise, not in the contest situation, but in other contexts, for example escape from predators, whereas the disadvantages of excessive persistence would arise from waste of time and energy in the contest itself. Third, the disadvantage of excessive persistence would be felt to the same extent by the ultimate winner as by the ultimate loser. For these reasons, the analysis which follows is relevant primarily to displays and not to tournaments. In real contests the distinction between a tournament and a display may not be as clear as I have suggested; the analysis will apply to the extent that the assumptions just given concerning the disadvantages of excessive persistence hold. $\uparrow$

Many complications which arise in actual contests between animals have been ignored. Perhaps the most important are the changes which may occur with age in an individual's chance of success, and the effects of previous experience of contests with the same opponent.

\section{Game Theory and Animal Contests}

The first attempt to apply the theory of games to evolution was made by Lewontin (1961). He was concerned with the evolution of genetic mechanisms, which he viewed as a game played between a species and nature. He argued that a species should adopt the "maximin" strategy - that is, the strategy which gives it the best chance of survival if nature does its worst. His approach was therefore very different from that adopted here; it tacitly assumed group selection, and the maximin strategy is not in general the same as the evolutionarily stable strategy.

† A similar distinction has been made independently by Parker (1974). His paper is mainly concerned with ritualized contests which do provide reliable information about the outcome of escalation, whereas this paper is concerned with ritualized contests which do not. 
Our own approach owes more to the papers by Hamilton (1967) and MacArthur (1965) on the evolution of the sex ratio. Hamilton pointed out that the choice by an individual of a sex ratio for its offspring can be seen as a choice of a strategy in a game against others, designed to maximize the individual's contribution to future generations. MacArthur (1965), although he did not explicitly refer to game theory, sought for a sex ratio which was evolutionarily stable in precisely the sense defined below.

A major difficulty in applying game theory to human conflicts lies in the need to place a numerical value, or "utility", on the preferences the players place on the possible outcomes. How for example does one put the utilities of financial reward and of injury or death on the same numerical scale? This difficulty does not arise, at least in principle, in applying game theory to animal behaviour. In human conflicts, strategies are chosen by reason to maximize the satisfaction of human desires-or at least it is in those terms that they are analyzed by game theorists. Strategies in animal contests are naturally selected to maximize the fitness of the contestants. Thus apparently incommensurable outcomes can be placed on a single scale of utility according to the contribution they make to reproductive success. This equivalence between utility and contribution to fitness is the main justification for applying game theory to animal contests.

The second general point is the nature of the "solution" we are seeking. If behaviour has evolved under individual selection, then the solution we want is an "evolutionarily stable strategy" or ESS. An ESS, which may be a pure or mixed strategy, is defined as follows. A strategy $I$ is an ESS if the expected utility of $I$ played against itself is greater than the utility of any other strategy $J$ played against $I$. This can be written

$$
E_{I}(I)>E_{I}(J)
$$

where $E$ gives the expected utility of the strategy in parentheses played against the strategy indicated by the subscript.

The relevance of this definition is as follows. In a population consisting entirely of individuals adopting strategy $I$, rare variants arising by mutation which adopted a different strategy $J$ would not increase in frequency, and hence the population would be stable under mutation and selection.

In the definition, we have required that $E_{I}(I)>E_{I}(J)$; difficulties arise if $E_{I}(I)=E_{l}(J)$. In this case, $I$ is an equilibrium strategy, but it need not be stable. To determine the stability, we need to know $E_{J}(J)$ and $E_{J}(I)$. Thus in a population of which a fraction $p$ adopt $I$ and $(1-p)$ adopt $J$, the expected "fitnesses" are

$$
\begin{aligned}
& E(I)=p E_{I}(I)+(1-p) E_{J}(I), \\
& E(J)=p E_{I}(J)+(1-p) E_{J}(J) .
\end{aligned}
$$

$I$ will then be evolutionarily stable if $E_{J}(I)>E_{J}(J)$. 
Thus we can extend our definition, and say that $I$ is an ESS if, for all alternative strategies $J$, either

$$
E_{I}(I)>E_{I}(J),
$$

or

$$
E_{I}(I)=E_{I}(J) \quad \text { and } \quad E_{J}(I)>E_{J}(J) .
$$

The stability need not be global. There may be more than one ESS for a given game. If so, a population would evolve to a different ESS according to its initial composition.

An ESS may be a "mixed" strategy; that is, it may consist of adopting one out of a set of "pure" strategies according to a set of preassigned probabilities. If so, a stable population could either be genetically polymorphic, with appropriate frequencies of individuals adopting different pure strategies, or it could be monomorphic, the behaviour of all individuals being random in an appropriate way.

In the appendix to this paper, Dr J. Haigh presents a preliminary analysis of the circumstances in which a game will have an ESS, and shows how one can search for an ESS if it exists. He considers games in which there is a finite number of pure strategies, and in which the pay-offs are the same for both players-i.e. if $A$ and $B$ are playing a game, the pay-off to $A$ if he plays $I$ and if $B$ plays $J$ is the same as the pay-off to $B$ if he plays $I$ and $A$ plays $J$. He shows that if there are only two pure strategies it is always possible to find an ESS. If there are three or more pure strategies, there may be no ESS. Games can be constructed with three pure strategies which have no ESS. A population playing such a game, with pay-offs determining contributions to the next generation, was simulated on a computer; it evolved cyclically without ever reaching an equilibrium. So far, however, games which we have thought of as being models of some biological process have proved to have at least one ESS.

\section{A Simple Model}

Consider a contest between two individuals in which victory goes to the contestant who is prepared to continue for longer. Suppose that the pay-off to the victor is $v$. If a contest is ever to be settled, there must also be some disadvantage to the contestants in a long contest. If so, the only choice of strategy open to a contestant is of the period for which he is prepared to continue, and hence of the pay-off, say $-m$, he is prepared to accept. Thus if two contestants adopt strategies $m_{1}$ and $m_{2}$, where $m_{1}>m_{2}$, the pay-off to the first is $v-m_{2}$ and to the second is $-m_{2}$. Our problem then is: how should a contestant choose a value of $m$, or, more precisely, is there a method of choosing $m$ which is an ESS? 
It is easy to see that no pure strategy is an ESS. Thus suppose that strategy $I$ selects $m_{1}+\varepsilon$, where $\varepsilon$ is a small random variable with mean zero; $\varepsilon$ is introduced to ensure that two contestants adopting $I$ have equal chances of winning. Then $E_{I}(I)=\frac{1}{2} v-m_{1}$. Strategy $J$ selects $m_{2}$. If $m_{2}>m_{1}$, then $E_{I}(J)=v-m_{1}$. Hence $E_{J}(I)>E_{I}(I)$, and $I$ cannot be an ESS.

This reasoning might suggest that a contestant should select the highest possible value of $m$ so as to make it difficult for his opponent to select a higher one. This is not so, because if $m_{1}>\frac{1}{2} v, E_{I}(I)<0$. Then if $m_{2}=0$, $E_{I}(J)=0$, and therefore $I$ is again unstable.

There is however a mixed strategy which is an ESS. This can be found as follows. Strategy $I$ is a mixed strategy which selects a value between $x$ and $x+\delta x$ with probability $p(x) \delta x$. Then the expected gain of playing a fixed value $m$ against $I$ is

$$
E(m)=\int_{0}^{m}(v-x) p(x) \mathrm{d} x-\int_{m}^{\infty} m p(x) \mathrm{d} x .
$$

We now choose the function $p(x)$ so that $E(m)$ has the same constant value $C$ for all values of $m$. Then $C$ is the expected pay-off of any pure strategy played against $I$. Since the expected pay-off of any mixed strategy played against $I$ is the weighted mean of the pay-offs of the pure strategies composing it, the expected gain of any mixed strategy played against $I$ is likewise $C$. By the same reasoning, $C$ is the pay-off of $I$ played against itself. Thus if we can find a function $p(x)$ such that $E(m)=C$, we shall have found an equilibrium strategy; we shall then show that the equilibrium is stable.

To find $p(x)$, we put $E(m)=E(m+\delta m)$, so that

$$
\begin{aligned}
\int_{0}^{m}(v-x) p(x) \mathrm{d} x-\int_{m}^{\infty} m p(x) \mathrm{d} x=\int_{0}^{m+\delta m}(v-x) p(x) \mathrm{d} x & \\
& -\int_{m+\delta m}^{\infty}(m+\delta m) p(x) \mathrm{d} x .
\end{aligned}
$$

After a little manipulation, remembering that $\int_{0}^{\infty} p(x) \mathrm{d} x=1$, this gives

$$
v p(m)=1-\int_{0}^{m} p(x) \mathrm{d} x .
$$

Equation (3) is satisfied by the function

$$
p(x)=\frac{1}{v} \mathrm{e}^{-x / n},
$$

which is the equilibrium strategy we are seeking. We have now to show that the equilibrium is stable. Thus if strategy $J$ plays a fixed value $m$, we have to show that $E_{J}(I)>E_{J}(J)$ for all values of $m$. 
Now

and

$$
E_{J}(J)=\frac{1}{2} v-m,
$$

Hence

$$
\begin{aligned}
E_{J}(I) & =(v-m) \int_{m}^{\infty} p(x) \mathrm{d} x-\int_{0}^{m} x p(x) \mathrm{d} x \\
& =2 v \mathrm{e}^{-m / v}-v .
\end{aligned}
$$

$$
E_{J}(I)-E_{J}(J)=2 v \mathrm{e}^{-m / v}-3 v / 2+m,
$$

which is positive for all positive values of $m$. Therefore the strategy defined by the distribution (4) is an ESS.

We conclude that an evolutionarily stable population is either genetically polymorphic, the strategies of individuals being distributed as in (4), or that it consists of individuals whose behaviour differs from contest to contest as in (4). There is no stable pure strategy, and hence no behaviourally uniform population can be stable.

Substituting $p(x)=1 / v \mathrm{e}^{-v / x}$ in (2), we find that $E(m)=0$. This may at first sight seem odd. Thus if we were to imagine that the reward for victory, $v$, is the obtaining of an item of food, and that the cost of the contest is the food equivalent of the energy used in the contest, it might seem that an evolutionarily stable population would use up in fighting all the energy it obtained from its food. Clearly no such population could survive. The absurdity arises because of a misinterpretation of the utility $v$. The advantage that the winner of such a contest has over the loser is to be measured not by the energy in the food obtained, but by the energy which the loser must expend in finding a second similar item of food. This point can be clarified by showing how the present model might apply to two other situations:

(1) Two birds attempt to establish a territory in an area too small to contain both. The loser will have to establish a territory in some other probably less favourable habitat. The pay-off for winning is therefore the difference between the expected reproductive success in the contested territory and reproductive success in the alternative habitat. The cost of continuing the contest, to both contestants, is the loss of reproductive success caused by the delay in starting to breed, the expenditure of energy on display, etc.

(2) Two individuals compete over the relative positions they shall occupy in a social hierarchy or peck order. The pay-off for winning is measured by the increased reproductive success consequent on being higher in the peck order; the cost of the contest is again measured by the expenditure of energy and any other risks that may be associated with a protracted contest.

There is therefore nothing absurd about the conclusion that $E(m)=0$. However, the ESS is not that which would be favoured by group selection. For the group, the optimal strategy is to continue for a very short random period. 


\section{Should a Contestant Give Information About His Intentions?}

It has been assumed in the preceding discussion that a contestant displays at full intensity until the predetermined moment when he retreats. Would it pay a contestant to convey to his opponent information about his intentions? In more biological terms, should the intensity of display be related to the length of time for which the contestant will continue? The short answer to this question is "no", but the problem deserves further consideration.

The first point to establish is that the information conveyed would have to be accurate. It is no use suggesting that each contestant should announce his intentions, and that then the contestant whose announced intentions are lower should at once retreat. In such a game, the only rational strategy would be to announce that one will go on for ever.

Given that the information conveyed is accurate, it is easy to see that it is of no selective advantage to convey it. Thus consider two possible strategies: $I$, who conveys no information about his intentions, but can modify his intentions in the light of information received; and $J$, who announces his intentions and cannot then modify them. At first sight it seems that if $J$ announces a value of $m$ greater than $v$, he is bound to win against $I$, because $I$ would be foolish to compete. This, however, is mistaken. Thus suppose I selects his strategy according to the distribution (4), and ignores the information he receives. Then it follows from the argument in the preceding section that $I$ is an ESS. A still more favourable strategy, $I^{\prime}$, would be to behave according to the distribution (4) if $J$ announced a strategy greater than $v$, but if $J$ announced a strategy less than $v$ to continue displaying until victory. It is then easy to show that there is no strategy $J$, pure or mixed, which is stable against $I^{\prime}$; that is, for which $E_{J}(J)>E_{J}\left(I^{\prime}\right)$. It follows that selection will oppose any tendency for the intensity of display to reveal future intentions.

In case it seems that the proposed strategy $I^{\prime}$ is too complex to evolve in actual animal species, it is worth pointing out that a genetically polymorphic population, in which (4) gives the frequency of individuals which always display for a period $x$, except in those cases when their opponent makes it clear that he does not intend to display for long, in which case the individual displays until his opponent retreats, is a realization of strategy $I^{\prime}$.

The predictions of this and the preceding sections, that the duration of contests should be very variable, and that an individual should display with constant intensity, independent of how much longer they will continue, needs experimental investigation. The most relevant investigation known to me is that of Simpson (1968) on the Siamese fighting fish Betta splendens. This agrees rather well with both predictions. Different contests do last very different lengths of time, and there are no statistically significant or consistent 
differences between the patterns of behaviour of the eventual winner and the eventual loser, at least until close to the end of a contest. It must be admitted that the present model gives no explanation for the variety of behavioural components observed in these contests.

\section{Contests with Asymmetrical Conditions}

A population of rational beings playing the game considered in section 3 would agree to toss a coin to decide each contest, the loser retreating at once. Is there any way, other than by group selection, in which a population of animals could adopt an equivalently satisfactory solution? For example, in contests for territory, if later arrivals always retreated in the face of an earlier occupant, this would provide a "rational" policy reducing the length of contests. The difficulty here is that if the chances of victory of later and earlier arrivals are equal, why should the former retreat? (In this particular case, chances might not be equal, because neighbours might attack a newcomer more vigorously than an established occupant, but this is a complication I shall ignore.) I shall now show that if there is associated with each contest an asymmetry (e.g. late $v$. early arrival) recognizable to both contestants, then this can lead to the evolution of a stable strategy comparable to coin-tossing, even if the asymmetry does not alter the probabilities of victory in a contest.

Thus suppose as before that the pay-off for winning is $v$, and the price to both contestants is measured by the duration of the contest. In addition, suppose that there is a "label" associated with each contest, so that one contestant "sees" the label A and the other the label B; in a series of contests, the frequencies with which any contestant sees $A$ and $B$ are equal. Does the presence of the label alter the nature of the ESS?

Consider strategy $I$, as follows:

$$
\begin{aligned}
& \text { if see A, play } M \text {, where } M>v \text {, } \\
& \text { if see } \mathrm{B} \text {, play } 0 \text {. }
\end{aligned}
$$

If the alternative to $I$ is strategy $J$, which plays a fixed $m$ regardless of the label, then

$$
\begin{aligned}
E_{I}(I) & =\frac{1}{2} v, \\
\text { if } m<M, \text { then } E_{I}(J) & =\frac{1}{2} v-\frac{1}{2} m, \\
\text { if } m>M, \text { then } E_{Y}(J) & =v-\frac{1}{2} M .
\end{aligned}
$$

Since $M>v, E_{I}(I)>E_{I}(J)$ for all positive values of $m$.

If $K$ is a mixed strategy which ignores the label, $E_{I}(K)$ is the weighted mean of a number of values of $E_{I}(J)$, and hence $E_{I}(I)>E_{I}(K)$. Hence $I$ is stable against any pure or mixed strategy which ignores the label. 
By symmetry, the strategy -if see A, play 0 ; if see B, play $M$-is also an ESS. Of course, these two symmetric strategies cannot co-exist. Whichever is first established will continue indefinitely. But what of the strategy $P$, which ignores the label and plays according to the distribution (4)? We have $E_{P}(P)=0$ and $E_{P}(I)=0$, so the stability of $P$ depends on the values of $E_{l}(I)$ and $E_{I}(P)$.

Now

and from equation (5),

$$
E_{\mathrm{I}}(I)=\frac{1}{2} v,
$$

$$
E_{I}(P)=\frac{1}{2} v+\frac{1}{2}\left(2 v \mathrm{e}^{-M / v}-v\right) .
$$

Since $M>v, E_{J}(P)<v / \mathrm{e}<E_{I}(I)$, so that $I$ is stable and $P$ unstable. This establishes our earlier assertion that there is an ESS comparable to the rational policy of coin-tossing.

In biological terms, a method of settling contests by taking into account some asymmetric feature, such as first arrival, which could not by itself influence the outcome, can be evolutionarily stable. A possible example has been suggested to me by $\operatorname{Dr} \mathrm{M}$. Land. Juvenile jumping spiders defend a region of space around where they happen to be. If two individuals meet, the first one to see his opponent orientates and advances towards him, and the other retreats at once without a contest. This is a trivial contest. A more important example of a contest being settled by an initial asymmetry is reported by Kummer (1971) in hamadryas baboons. In the wild or in captivity, two males may fight over the possession of a female. Kummer brought together a male and female previously strange to one another, and left them for $15 \mathrm{~min}$ to form a pair bond. A second male, who had been able to observe the pair, was then introduced into the enclosure; this second male avoided conflict with the first arrival, who was left in possession of the female. On a later occasion the same two males were used in a similar experiment with a second female, but with their roles reversed; again the first arrival was left in possession of the female. Although the argument of this section applies to displays rather than to actual fights, the logic is the same provided that if a fight takes place both participants run a risk of injury. Kummer's experiment is a clear illustration of the fact that an initial asymmetry, which is completely irrelevant to the outcome of a fight, can be used to settle a contest.

\section{REFERENCES}

GeIST, V. (1966). Behaviour 27, 175.

Hamil ToN, W. D. (1964). J. theor. Biol. 7, 1.

Hamaton, W. C. (1967). Science, N.Y. 156, 147.

Hammton, W. C. (1971). In Man and Beast: Comparative Social Behaviour (J. F. Eisenberg \& W. S. Dillon, eds). Washington: Smithsonian Press.

HuXley, J. S. (1956). Phil. Trans. R. Soc. Ser. B 251, 249. 
Kummer, H. (1971). Primate Societies. Chicago: Aldine-Atherton.

LEWONTIN, R. C. (1961). J. theor. Biol. 1, 382.

MACARTHUR, R. H. (1965). In Theoretical and Mathematical Biology (T. Waterman \& H. Horowitz, eds). New York: Blaisdell.

MAYNARd Smith, J. (1971). J. theor. Biol. 30, 319.

Maynard Smith, J, \& Price, G. R. (1973). Nature, Lond. 246, 15.

PARKER, G. A. (1974). J. theor. Biol. 47, 223.

Simpson, M. J. A. (1968). Animal Behaviour Monographs 1, 1.

Trivers, R. L. (1972). In Sexual Selection and the Descent of Man (B. Campbell, ed.). London: Heinemann.

Williams, G. C. (1966). Adaptation and Natural Selection. Princeton: Princeton University Press.

Williams, G. C. \& Mitton, J. B. (1973). J. theor. Biol. 39, 545.

WyNne-EDWARDS, V. C. (1962). Animal Dispersion in Relation to Social Behaviour.

Edinburgh: Oliver and Boyd.

\section{APPENDIX \\ The Existence of Evolutionary Stable Strategies}

JoHn HaIGH

\section{Mathematics Division, University of Sussex}

Consider a situation where $a_{i j}(i, j=1,2, \ldots, m)$ is the pay-off to the player of strategy $i$ when his opponent plays strategy $j$ in a game of $m$ pure strategies. Then any probability vector

$$
\mathbf{p}=\left(p_{1}, \ldots, p_{m}\right)\left(\sum_{i=1}^{m} p_{i}=1, p_{i} \geqslant 0 \text { for } i=1,2, \ldots, m\right)
$$

represents the mixed strategy

"Play the $i$ th pure strategy with probability $p_{i}, 1 \leqslant i \leqslant m$ ".

Then, if $E(\mathbf{p}, \mathbf{q})$ is the mean pay-off to a player who uses $p$ when his opponent uses $\mathbf{q}$, we have

$$
E(\mathbf{p}, \mathbf{q})=\mathbf{p}^{\prime} \mathbf{A q}
$$

where $\mathbf{A}$ is the matrix $\left(a_{i j}\right)$ and $\mathbf{p}$ is a column vector, $\mathbf{p}^{\prime}$ its (row) transpose.

According to the definition in the paper, $p$ is an ESS (evolutionarily stable strategy) provided

$$
E(\mathbf{p}, \mathbf{p}) \geqslant E(\mathbf{q}, \mathbf{p}) \text { for all } \mathbf{q}
$$

and, if $\mathbf{p} \neq \mathbf{q}$ but, $E(\mathbf{p}, \mathbf{p})=E(\mathbf{q}, \mathbf{p})$, then

$$
E(\mathbf{p}, \mathbf{q})>E(\mathbf{q}, \mathbf{q}) \text {. }
$$

It seems a natural conjecture, by analogy with game theory, to suggest that, whatever the matrix $\mathbf{A}$, there is some ESS. There are some trivial cases 
we should exclude-for example, if $a_{i i}=a_{i j}=a_{j i}=a_{j j}$, then any strategy p that has only $p_{i}, p_{j}>0$, played against any strategy $q$ that has only $q_{i}, q_{j}>0$ will clearly result in the same value of $E(\mathbf{p}, \mathbf{p}), E(\mathbf{p}, \mathbf{q})$ and $E(\mathbf{q}, \mathbf{q})$, so the strict inequality in equation (A2) cannot arise. However, even leaving aside such trivial cases, [or even relaxing equation (A2) so that if $E(\mathbf{p}, \mathbf{p})=E(\mathbf{q}, \mathbf{p})$, we only require $E(\mathbf{p}, \mathbf{q}) \geqslant E(\mathbf{q}, \mathbf{q})]$ the conjecture is false. There are non-trivial situations where no ESS exists. However, we will first give sufficient conditions on $\mathbf{A}$ that do guarantee the existence of an ESS in certain circumstances.

Theorem 1: If, for any $i, a_{i i}>a_{j i}$ for all $j \neq i$, then $i$ is a pure ESS.

Proof: Obvious from the definition.

This case can be best described as the "diagonally dominant case"-- A has a column in which the term on the diagonal dominates all other terms. It is quite possible that $\mathbf{A}$ may have several diagonally dominant columns, and thus several different pure ESS's.

Theorem 2: If $\mathbf{A}$ is a non-trivial $2 \times 2$ matrix, there is an FSS.

Proof: We may suppose

$$
\mathbf{A}=\left(\begin{array}{ll}
0 & b \\
a & c
\end{array}\right)
$$

where $a \geqslant 0$ and $b \geqslant c$. (For, given any $\mathbf{A}$, to subtract amount $a_{11}$ from each pay-off merely decreases evenly the reward, and does not change the relative merits of the strategies. And, if $a<0$ or $b<c$, there is already an ESS by Theorem 1.)

Then it can be verified that $(p, 1-p)$ is an ESS, where

$$
p=\frac{b-c}{a+b-c}
$$

if $b=c$ and $a=0$, so that this expression is indeterminate, then

$$
\mathbf{A}=\left(\begin{array}{ll}
0 & b \\
0 & b
\end{array}\right)
$$

so here the pay-off is independent of your own strategy, which we again consider a trivial case. For $3 \times 3$ or larger matrices, some preliminary work is helpful

$$
\left(\mathbf{p}^{\prime}-\mathbf{q}^{\prime}\right) \mathbf{A}(\mathbf{p}-\mathbf{q})=\left(\mathbf{p}^{\prime} \mathbf{A p}-\mathbf{q}^{\prime} \mathbf{A p}\right)-\left(\mathbf{p}^{\prime} \mathbf{A q}-\mathbf{q}^{\prime} \mathbf{A q}\right) .
$$

Hence, if $p^{\prime} \mathbf{A p}=q^{\prime} \mathbf{A p}$, we shall require (for an ESS) $p^{\prime} \mathbf{A q}>q^{\prime} \mathbf{A q}$, which means that, if $\mathbf{p}^{\prime} \mathbf{A p}=\mathbf{q}^{\prime} \mathbf{A p}$, then $\left(\mathbf{p}^{\prime}-\mathbf{q}^{\prime}\right) \mathbf{A}(\mathbf{p}-\mathbf{q})<\mathbf{0}$. If $\boldsymbol{A}$ were a positive definite matrix, this could not be satisfied, so to find a counterexample to the conjecture, we look for positive definite matrices without any diagonally dominant column. 
Now $2(x+y+z)^{2}+(x+y)^{2}+(x+z)^{2}$ can be written

and so the matrix

$$
\left(\begin{array}{lll}
x & y & z
\end{array}\right)\left(\begin{array}{lll}
4 & 1 & 5 \\
5 & 3 & 0 \\
1 & 4 & 3
\end{array}\right)\left(\begin{array}{l}
x \\
y \\
z
\end{array}\right)
$$

$$
\mathbf{B}=\left(\begin{array}{lll}
4 & 1 & 5 \\
5 & 3 & 0 \\
1 & 4 & 3
\end{array}\right)
$$

looks a candidate for a counterexample.

Theorem 3: A necessary condition for $\mathbf{p}$ to be an ESS is that when $\mathbf{p}_{i}>0$, then $(\mathrm{A} p)_{i}=\operatorname{Max}_{j}(\mathrm{~A} p)_{j}$.

Proof: Since $\mathbf{p}^{\prime} \mathbf{A p} \geqslant \mathbf{q}^{\prime}$ Ap for all $\mathbf{q}$, consider the problem of maximizing (as $\mathbf{r}$ varies with $\mathbf{p}$ fixed) $\mathbf{r}^{\prime} \mathbf{A p}$.

Suppose

$$
\mathbf{r}^{\prime} \mathbf{A p}=\sum_{j} r_{j}(\mathbf{A p})_{j}
$$

$$
p_{i}>0 \text { but }(\mathbf{A p})_{i}<\underset{j}{\operatorname{Max}}(\mathbf{A p})_{j}=(\mathbf{A p})_{k} \text {. }
$$

Then, if $\mathbf{r}$ is the $k$ th pure strategy, $\mathbf{r}^{\prime} \mathbf{A p}=(\mathbf{A p})_{k}$ and

$$
\mathbf{p}^{\prime} \mathbf{A p}=p_{i}(\mathbf{A p})_{i}+\sum_{j \neq i} p_{j}(\mathbf{A p})_{j}<(\mathbf{A p})_{k}
$$

since $p_{i}>0$. It is an immediate consequence of Theorem 3 (and it is also clear after a brief thought) that no pure strategy $i$ can be an ESS if $a_{i i}<a_{j i}$ for some $j$ (a converse to Theorem 1).

Thus no pure strategy of the matrix $\mathbf{B}$ is an ESS. Suppose $(0, p, 1-p)$ $(1>p>0)$ is an ESS. By Theorem 3, we must have $4 p+3(1-p)=3 p+0(1-p)$ i.e. $2 p-3=0$, impossible. Similarly, for $(p, 0,1-p)$ to be an ESS $p+3(1-p)=4 p+5(1-p)$ i.e. $p+2=0$, impossible. And, for $(p, 1-p, 0)$, $4 p+(1-p)=5 p+3(1-p)$ i.e. $p=2$, impossible. For $(p, q, 1-p-q)$ $[0<p, q, 1-p-q<1]$ to be an ESS

$$
4 p+q+5(1-p-q)=5 p+3 q=p+4 q+3(1-p-q)
$$

i.e.

$$
(p, q, 1-p-q)=\left(\frac{11}{37}, \frac{17}{37}, \frac{9}{37}\right)
$$

and any strategy played against that has an expectation of $\frac{106}{37}$. But, playing $\left(\frac{1}{37}, \frac{17}{37}, \frac{9}{37}\right)$ against the opponent's $(0,0,1)$ gains $\frac{82}{37}$, but playing $(0,0,1)$ against $(0,0,1)$ gains 3 , and $3>\frac{82}{37}$. Thus no ESS can exist.

This example shows that the condition of Theorem 3 is not sufficient for finding an ESS. But it also shows a logical way of seeking out all possible ESS's. 